\title{
The Quest for Type 2 Diabetes Subgroups Identification: Literature Review for a New Subtype Proposal
}

\author{
Mohamed Islam Delma ${ }^{1}$ \\ 1. Internal Medicine, Colonel Chaabani Hospital, El Menia, DZA
}

Corresponding author: Mohamed Islam Delma, mohamed.islam.delma@gmail.com

\begin{abstract}
Type 2 diabetes is considered typically as a heterogeneous disease that englobes the potential different subtypes with distinct pathophysiological mechanisms and/or susceptibility to complications. Some authors have succeeded in the identification of some of these subgroups, but a lot of work remains to be completed. Given the effects of the sympathetic innervation via alpha 1 adrenoceptors on diabetes target organs and the interindividual variability of this receptor sensitivity, the existence of a subtype of type 2 diabetes with hyperactivation of alpha 1 adrenoceptors (HA1A) is proposed. Based on the literature review, the potential characteristics of this phenotype and its susceptibility to certain complications have been identified in this article.
\end{abstract}

Categories: Endocrinology/Diabetes/Metabolism, Internal Medicine, Neurology

Keywords: type 2 diabetes, alpha 1 adrenoreceptor, silent myocardial infarction, diabetic nephropathy, sympathetic innervation, diabetes complications, diabetic neuropathy

\section{Introduction And Background}

Diabetes mellitus is a metabolic disorder characterized by hyperglycemia. The diagnosis is established based on well-defined biologic criteria. Type 2 accounts for $90 \%$ to $95 \%$ of all diabetes and is caused by insulin deficiency associated with insulin resistance [1]. It has become clear that type 2 diabetes is a heterogeneous disease englobing different groups with distinct physiopathology, evolution, and susceptibility to complications [2-3]. These subgroups may be defined by either a common pathophysiological mechanism or susceptibility to specific complications. Identifying these groups seems to be the gateway for a physiopathology-based therapeutic approach and complication-oriented screening for timely and better management. In fact, much effort has been made in this direction. Li and his colleagues have distinguished three subtypes associated with a distinct type of complications [3], while the study carried by Ahlqvist et al. identified five clusters of patients with a different pattern of complications [2].

Received 10/04/2018 Review began 10/09/2018 Review ended 12/20/2018 Published 12/24/2018

๑) Copyright 2018 Delma. This is an open access article distributed under the terms of the Creative Commons Attribution License CC-BY 3.0., which permits unrestricted use, distribution, and reproduction in any medium, provided the original author and source are credited.
Long ago, the pathogenic role of the sympathetic innervation via adrenoceptors in hyperglycemia had been established [4-5]. Of note, an interindividual variability of alpha 1 adrenoceptor subtype activity had also been reported [6].

Based on this background, this study developed a hypothesis of the existence of a type 2 diabetes subtype with hyperactivation of the alpha 1 adrenoceptors (HA1A).

\section{Review}

\section{Overview of alpha 1 adrenoceptors}

\section{Generality}

Based on the original research studies, Calzada et al. have provided a systematic review of the adrenoceptor subtypes [7]. In summary, adrenoceptors are membrane receptors responsible for mediating responses to the endogenous catecholamines including noradrenaline and adrenaline. Initially, they were classified as alpha receptors with preponderant excitatory effects and beta receptors with marked inhibitory effects. Subsequently, subtypes for alpha and beta receptors were discovered. Alpha receptors were subdivided into alpha 1 and alpha 2, based on their pharmacologic proprieties. Subsequently, with the advancement of pharmacologic and cloning techniques, subdivisions of alpha 1 and alpha 2 were established. Alpha 1 adrenoceptors are subdivided into alpha 1a, alpha 1b, and alpha $1 \mathrm{~d}$ subtypes, but their distinct distributions and effects are not fully understood. Hence, in this article, they have been referred to as alpha 1 adrenoceptors.

\section{Alpha 1 Adrenoceptor Activity Evaluation}

A method for the evaluation of alpha 1 adrenoceptor activity is via the assessment of human dorsal hand 
vein alpha-receptor activity [6]. The amplitude of dorsal hand vein vasoconstriction induced by alpha 1 agonist phenylephrine is measured by a device called the linear variable differential transformer. The dose of the alpha 1 agonist that induces $50 \%$ of vasoconstriction is used to compare individual sensitivity of alpha 1 adrenoceptors [6].

Another method consists of measuring the dose of phenylephrine required to elevate the mean arterial pressure by $25 \mathrm{mmHg}$ [8].

The results of these techniques have confirmed the interindividual variability of alpha 1 adrenoceptor sensitivity [6,9]. In the next section, the characteristics of type 2 patients with HA1A have been proposed.

\section{Characteristics of type 2 diabetes with alpha 1 receptor hyperactivation}

Demographic Parameters

Although some authors have found that some parameters are associated with alpha 1

adrenoceptor sensitivity such as sex and ethnicity, these findings were not consistent among all studies [9].

Anxiety

If we refer to the role of sympathetic innervation on anxiety and the experimental findings of the involvement of the alpha 1 adrenoceptors in stress-induced anxiety-like responses [10-11], we may expect that anxiety would be a trait of patients with this subtype.

\section{Diabetogenic Effect}

If the two mechanisms unanimously incriminated in type 2 diabetes and the defects in insulin secretion and exaggerated insulin resistance are considered alone, alpha 1 adrenoceptor hyperactivation would have only a mild diabetogenic effect, as its effect on these two mechanisms is only moderate and/or inconsistent among studies [12-13]. But if the role of glucagon on type 2 diabetes is taken into account [14], the effect of HA1A would be important, as this receptor induces glucagon secretion and potentiates the activity of adenylyl cyclase, a mediator of the glucagon receptor signaling pathway [15-16]. Overall, the development of type 2 diabetes in individuals with HA1A would be conditioned by the presence of an additional predisposing factor.

Due to the sympathetic system activation in the postprandial period [17], the characteristic postprandial glycemic profile in patients with HA1A subtype would be an accentuation of the postprandial hyperglycemia, followed by a relative decrease in the glycemic value. The first effect would be due to the potentiation of postprandial glucagon-induced hyperglycemia, while the second would be caused by the increase in glucagon effect on insulin secretion and is dependent on insulin reserve [18-19]. Furthermore, this profile may be present even in prediabetes, as it would be attested in glucose tolerance test (HGPO).

Lipidic Profile

Pool and his colleagues have demonstrated that alpha 1 receptor blockade induces a rise in high-density lipoprotein (HDL) levels and a reduction in low-density lipoprotein (LDL) levels and apolipoprotein B (apo B) [20]. This suggests that alpha 1 adrenoceptor stimulation may exercise the opposite effect. The patients with type 2 diabetes with HA1A are expected to have a thrombogenic lipidic profile with low HDL levels and high LDL and apo B levels. If this assumption is confirmed, the outcomes of alpha 1 adrenoceptor blockade in this subgroup, especially in patients with the highest cardiovascular risk, should be evaluated.

Concerning triglyceridemia, patients with HA1A subtype may have a tendency to hypertriglyceridemia. This is expected due to the hepatic alpha 1 adrenoceptor role in triglyceride liberation from the liver [21-22].

Silent Myocardial Infarction

Silent myocardial infarction (SMI) is a myocardial ischemic event without pain. Systematic screening for this condition by stress tests in diabetic patients is subject to debate [23]. Some authors advocate the screening program, given the high prevalence of cardiovascular diseases and SMI in patients with diabetes and the possible complications of undetected myocardial ischemia. Others argue against it, as there is not yet clear evidence of benefits from this systematic screening and consequent invasive therapeutics compared to good medical control of cardiovascular risk factors. But, how about patients with HA1A? Given the opposition of alpha 2 receptor analgesic effect by alpha 1 receptor [24] and the role of the former on SMI [25], myocardial infarction is more likely to be symptomatic than asymptomatic in diabetic patients with HA1A. If confirmed, this argument would advocate against systematic screening for SMI in HA1A subtype. 
The effect of sympathetic innervation via alpha 1 adrenergic receptors on penis detumescence through corpus cavernosum contraction is well-known [26]. This is the rationale of alpha 1 receptor blocker use in erectile dysfunction [26-27]. It is expected that patients with HA1A subtype would be more prone to this diabetes complication. A trial of the effect alpha 1 blocker on these patients should be evaluated via clinical trials.

Painful Neuropathy

The role of sympathetic innervation in this complication was suggested by the findings of a higher level of plasma norepinephrine in painful than in painless diabetic neuropathy [28]. The role of alpha 1

adrenoceptors seems to be primordial since it has been shown that nerve damage induces the upregulation of alpha 1 adrenoceptors that are responsible for exaggerating the adrenergic component of pain [29]. Patients with HA1A subtype are more likely to express nerve damage caused by chronic hyperglycemia as painful than painless neuropathy.

Another diabetes-related neuropathy is treatment-induced neuropathy, also known as insulin neuritis. It is defined as a painful, autonomic neuropathy that develops in the setting of rapid improvements in glycemic control in individuals with a long history of hyperglycemia [30]. The physiopathology is not completely clear, and some authors suggest a neural damage mechanism [31]. If this suggestion is validated, we may expect a susceptibility of HA1A subtype to this treatment-induced neuropathy.

Diabetic Retinopathy

Diabetic retinopathy is a microvascular complication of diabetes. Du et al. had shown, in their experimental study, that the alpha 1 adrenoceptor blockers inhibit the important features of diabetic retinopathy such as superoxide generation, proinflammatory protein expression, and the degeneration of retinal capillaries [32]. If their results are replicated in clinical studies, we expect that the blockade of alpha 1 adrenoceptors will be more beneficial in diabetic patients with HA1A.

Diabetic Nephropathy

Alpha 1 adrenoceptor autoantibodies have been objectified in some patients with diabetic nephropathy, with deleterious effects on kidney and arterial pressure [33-34]. These autoantibodies seem to be of stimulatory nature, as their effects are contracted by the alpha 1 adrenoceptor antagonists [33-34]. This led to the suggestion that HA1A may be harmful to the kidney. Hence, the clinical outcomes of alpha 1 adrenoceptor blockade on diabetic nephropathy prevention or regression should be evaluated in these type 2 patients with HA1A.

In summary, this paper aimed to develop the hypothesis of the existence of type 2 diabetes with HA1A subgroup, characterized by a common diabetogenic mechanism via glucagon synergism, and more importantly, a specific pattern of complications. Eventually, patients with this subtype may need an additional therapeutic option consisting of alpha 1 adrenoceptor blockade. This hypothesis is based on the literature review of different alpha 1 receptor effects, notably on diabetes target organs. However, some limitations should be considered. First, the aforementioned tests of alpha 1 receptor sensitivity evaluation may be specific of only one subtype of these receptors [9]. Given the lack of dispensible tests for all alpha 1 receptor subtypes, it is postulated in this paper that hyperactivation of one of them may reflect hyperactivation of the whole family. Eventually, if specific tests are developed, this suggestion should be reevaluated. Secondly, the existence of additional diabetes risk factors for each patient with HA1A is suggested, but we have omitted the potential influence of these factors on the evolution pattern of this subtype. This is helpful initially for a better characterization of this subtype, but these factors impact should be discussed later in a case per case basis.

\section{Conclusions}

Nowadays, type 2 diabetes is considered as a heterogeneous group encompassing different phenotypes. Identification of these phenotypes is the cornerstone of the personalized management of type 2 diabetes advocated by scientific societies. This paper aimed to identify the characteristics of a potential new subtype: type 2 diabetes with HA1A. Clinical studies are needed for confirmation or invalidation of this hypothesis.

\section{Additional Information}

\section{Disclosures}

Conflicts of interest: In compliance with the ICMJE uniform disclosure form, all authors declare the following: Payment/services info: All authors have declared that no financial support was received from any organization for the submitted work. Financial relationships: All authors have declared that they have no financial relationships at present or within the previous three years with any organizations that might 
have an interest in the submitted work. Other relationships: All authors have declared that there are no other relationships or activities that could appear to have influenced the submitted work.

\section{References}

1. American Diabetes Association: 2. Classification and diagnosis of diabetes: standards of medical care in diabetes-2018. Diabetes Care. 2018, 41: S13-S27. 10.2337/dc18-S002

2. Ahlqvist E, Storm P, Käräjämäki A, et al.: Novel subgroups of adult-onset diabetes and their association with outcomes: a data-driven cluster analysis of six variables. Lancet Diabetes Endocrinol. 2018, 6:361-369. 10.1016/S2213-8587(18)30051-2

3. Li L, Cheng WY, Glicksberg BS, et al.: Identification of type 2 diabetes subgroups through topological analysis of patient similarity. Sci Transl Med. 2015, 7: 311ra174. 10.1126/scitranslmed.aaa9364

4. Bruce DG, Chisholm DJ, Storlien LH, Kraegen EW, Smythe GA: The effects of sympathetic nervous system activation and psychological stress on glucose metabolism and blood pressure in subjects with type 2 (noninsulin-dependent) diabetes mellitus. Diabetologia. 1992, 35:835-43. 10.1007/BF00399929

5. Robertson RP, Halter JB, Porte D Jr: A role for alpha-adrenergic receptors in abnormal insulin secretion in diabetes mellitus. J Clin Invest. 1976, 57:791-795. 10.1172/JCI108338

6. Eichler HG, Ford GA, Blaschke TF, Swislocki A, Hoffman BB: Responsiveness of superficial hand veins to phenylephrine in essential hypertension. Alpha adrenergic blockade during prazosin therapy. J Clin Invest. 1989, 83:108-12. 10.1172/JCI113845

7. Civantos Calzada B, Aleixandre de Artiñano A: Alpha-adrenoceptor subtypes. Pharmacol Res. 2001, 44:195208. 10.1006/phrs.2001.0857

8. Sherwood A, Hill LK, Blumenthal JA, Johnson KS, Hinderliter AL: Race and sex differences in cardiovascular $\alpha$-adrenergic and $\beta$-adrenergic receptor responsiveness in men and women with high blood pressure. J Hypertens. 2017, 35:975-981. 10.1097/HJH.0000000000001266

9. Sofowora GG, Dishy V, Landau R, et al.: Alpha 1A-adrenergic receptor polymorphism and vascular response . Clin Pharmacol Ther. 2004, 75:539-45. 10.1016/j.clpt.2004.02.006

10. Pohjavaara P, Telaranta T, Väisänen E: The role of the sympathetic nervous system in anxiety: Is it possible to relieve anxiety with endoscopic sympathetic block?. Nord J Psychiatry. 2003, 57:55-60. $10.1080 / 08039480310000266$

11. Cecchi M, Khoshbouei H, Morilak DA: Modulatory effects of norepinephrine, acting on alpha 1 receptors in the central nucleus of the amygdala, on behavioral and neuroendocrine responses to acute immobilization stress. Neuropharmacology. 2002, 43:1139-47. 10.1016/S0028-3908(02)00292-7

12. Skoglund G, Lundquist I, Ahrén B: Effects of alpha 1- and alpha 2-adrenoceptor stimulation and blockade on plasma insulin levels in the mouse. Pancreas. 1986, 1:415-20.

13. McCarty MF: Elevated sympathetic activity may promote insulin resistance syndrome by activating alpha-1 adrenergic receptors on adipocytes. Med Hypotheses. 2004, 62:830-8. 10.1016/j.mehy.2003.11.007

14. Hædersdal S, Lund A, Knop FK, Vilsbøll T: The role of glucagon in the pathophysiology and treatment of type 2 diabetes. Mayo Clin Proc. 2018, 93:217-239. 10.1016/j.mayocp.2017.12.003

15. Vieira E, Liu YJ, Gylfe E: Involvement of alpha1 and beta-adrenoceptors in adrenaline stimulation of the glucagon-secreting mouse alpha-cell. Naunyn Schmiedebergs Arch Pharmacol. 2004, 369:179-83. 10.1007/s00210-003-0858-5

16. Karkanias GB, Petitti N, Etgen AM: Progesterone attenuation of alpha 1-adrenergic receptor stimulation of phosphoinositol hydrolysis in hypothalamus of estrogen-primed female rats. Endocrinology. 1995, 136:1993-9. 10.1210/endo.136.5.7720647

17. van Baak MA: Meal-induced activation of the sympathetic nervous system and its cardiovascular and thermogenic effects in man. Physiol Behav. 2008, 94:178-86. 10.1016/j.physbeh.2007.12.020

18. Henkel E, Menschikowski M, Koehler C, Leonhardt W, Hanefeld M: Impact of glucagon response on postprandial hyperglycemia in men with impaired glucose tolerance and type 2 diabetes mellitus. Metabolism. 2005, 54:1168-73. 10.1016/j.metabol.2005.03.024

19. Song G, Pacini G, Ahrén B, D'Argenio DZ: Glucagon increases insulin levels by stimulating insulin secretion without effect on insulin clearance in mice. Peptides. 2017, 88:74-79. 10.1016/j.peptides.2016.12.012

20. Pool JL, Lenz ML, Taylor AA: Alpha 1-adrenoreceptor blockade and the molecular basis of lipid metabolism alterations. J Hum Hypertens . 1990, 4:23-33.

21. Nonogaki K, Moser AH, Feingold KR, Grunfeld C: Alpha-adrenergic receptors mediate the hypertriglyceridemia induced by endotoxin, but not tumor necrosis factor, in rats. Endocrinology. 1994, 135:2644-50. 10.1210/endo.135.6.7988454

22. López-Soriano J, Carbó N, Costelli P, López-Soriano FJ, Argilés JM: $\alpha$-Adrenergic receptors may contribute to the hypertriglyceridemia associated with tumour growth. Cancer Lett. 1996, 110:213-6. 10.1016/S03043835(96)04501-6

23. Draman MS, Thabit H, Kiernan TJ, O'Neill J, Sreenan S, McDermott JH: A silent myocardial infarction in the diabetes outpatient clinic: case report and review of the literature. Endocrinol Diabetes Metab Case Rep. 2013, 2013:Accessed: December 24, 2018: https://edm.bioscientifica.com/view/journals/edm/2013/1/EDM13-0058.xml. 10.1530/EDM-13-0058

24. Gil DW, Cheevers CV, Kedzie KM, Manlapaz CA, Rao S, Tang E, Donello JE: Alpha-1-adrenergic receptor agonist activity of clinical alpha-adrenergic receptor agonists interferes with alpha-2-mediated analgesia. Anesthesiology. 2009, 110:401-7. 10.1097/ALN.0b013e3181943226

25. Chen $\mathrm{OJ}$, Lu L, Jin C, et al.: Insertion/insertion genotype of $\alpha(2 \mathrm{~B})$-adrenergic receptor gene polymorphism is associated with silent myocardial ischemia in patients with type 2 diabetes mellitus. Clin Biochem. 2010, 43:1201-4. 10.1016/i.clinbiochem.2010.07.023

26. Traish A, Kim NN, Moreland RB, Goldstein I: Role of alpha adrenergic receptors in erectile function . Int J Impot Res. 2000, 12:S48-63. 10.1038/sj.ijir.3900506

27. van Moorselaar RJ, Hartung R, Emberton M, et al.: Alfuzosin $10 \mathrm{mg}$ once daily improves sexual function in men with lower urinary tract symptoms and concomitant sexual dysfunction. BJU Int. 2005, 95:603-8. 


\section{Cureus}

10.1111/j.1464-410X.2005.05347.x

28. Tsigos C, Reed P, Weinkove C, White A, Young RJ: Plasma norepinephrine in sensory diabetic polyneuropathy. Diabetes Care. 1993, 16:722-7. 10.2337/diacare.16.5.722

29. Drummond PD: Neuronal changes resulting in up-regulation of alpha-1 adrenoceptors after peripheral nerve injury. Neural Regen Res. 2014, 9:1337-40. 10.4103/1673-5374.137583

30. Gibbons CH, Freeman R: Treatment-induced diabetic neuropathy: a reversible painful autonomic neuropathy. Ann Neurol. 2010, 67:534-41. 10.1002/ana.21952

31. Tesfaye S, Malik R, Harris N, Jakubowski JJ, Mody C, Rennie IG, Ward JD: Arterio-venous shunting and proliferating new vessels in acute painful neuropathy of rapid glycaemic control (insulin neuritis). Diabetologia. 1996, 39:329-35. 10.1007/BF00418349

32. Du Y, Cramer M, Lee CA, et al.: Adrenergic and serotonin receptors affect retinal superoxide generation in diabetic mice: relationship to capillary degeneration and permeability. FASEB J. 2015, 29:2194-204. 10.1096/fj.14-269431

33. Zhao LS, Xu CY: Effect of prazosin on diabetic nephropathy patients with positive $\alpha 1$-adrenergic receptor autoantibodies and refractory hypertension. Exp Ther Med. 2015, 9:177-182. 10.3892/etm.2014.2036

34. Zhao LS, Lin YY, Liu Y: Doxazosin attenuates renal matrix remodeling mediated by anti- $\alpha 1$-adrenergic receptor antibody in a rat model of diabetes mellitus. Exp Ther Med. 2017, 14:2543-2553.

10.3892/etm.2017.4827 